\title{
Biochemical diagnosis of phaeochromocytoma: Two instructive case reports
}

\author{
M F Stewart, P Reed, C Weinkove, K J Moriarty, A J Ralston
}

\begin{abstract}
The biochemical features of two patients with phaeochromocytomas illustrate the inadvisability of depending on a single group of analytes for the diagnosis. The first case presented as a surgical emergency with retroperitoneal haemorrhage. Biochemical diagnosis was difficult since total 24 hour urinary free catecholamine excretion was within normal limits in two out of three samples, and only marginally raised in the third with an atypical preponderance of adrenaline. Plasma catecholamine concentrations were also normal. But urinary excretion of the catecholamine metabolites, metadrenaline and 4-hydroxy-3-methoxy mandelic acid (HMMA), was consistently raised. In contrast, the second patient presenting with headache and labile hypertension showed normal metabolite excretion in the face of grossly increased free noradrenaline excretion and raised plasma noradrenaline concentrations.

It is therefore recommend that, as well as urinary free catecholamines, one group of their main metabolites, the 3-methoxy amines (normetadrenaline and metadrenaline) or HMMA, should routinely be measured whenever a phaeochromocytoma is suspected.
\end{abstract}

(F Clin Pathol 1993;46:280-282)

Phaeochromocytoma is an important diagnosis to make, because it is due to a benign, curable tumour in $90 \%$ of cases. ${ }^{1}$ Although rare in the general population, the incidence in hypertensive patients has been estimated to be between 1 in 1000 and 1 in $2500 .^{23}$ Diagnosis is difficult and major reliance is placed on biochemical investigation for confirmation and on expensive imaging techniques for localisation of the tumour. There is as yet no consensus on the best single biochemical test, but measurement of 24 hour urinary free (unconjugated) catecholamines (noradrenaline, adrenaline, and dopamine), using high performance liquid chromatography (HPLC) with electrochemical detection, has been recommended as a suitable first line investigation. ${ }^{4}$ This is mainly because it overcomes the problems of drug and dietary interference encountered with earlier assays of either 4-hydroxy-3-methoxy mandelic acid (HMMA, formerly known as vanillyl mandelic acid or VMA) and the 3-methoxy amine metabolites, normetadrenaline and metadrenaline.

We report two cases of phaeochromocytoma in which biochemical tests were falsely negative, forcing us to alter our investigative protocol.

\section{Case 1}

A 45 year old woman was investigated for palpitations and episodic breathlessness. She was normotensive with normal thyroid function tests and diagnosed as suffering from anxiety.

Four years later she was admitted to hospital with a sudden onset of central abdominal pain and vomiting. On examination she was apyrexial, pale, and distressed. She had a sinus tachycardia (100-112/minute), hypertension $(196 / 102 \mathrm{~mm} \mathrm{Hg})$, with noticeable tenderness and guarding on the right side of her abdomen and in the right renal angle. Emergency laparotomy showed a massive retroperitoneal haematoma extending over the right kidney, the second and third parts of the duodenum, and behind the ascending colon. She remained hypertensive $(170 / 100$ $\mathrm{mm} \mathrm{Hg}$ ) after surgery and an ultrasound scan indicated a mass in the right adrenal gland. The diagnosis of phaeochromocytoma was considered for the first time. Twenty four hour urinary free catecholamines and their metabolites, as well as plasma catecholamines, were measured by HPLC, as described previously. ${ }^{5}$ Results are shown in tables 1 and 2 .

The finding on a computed tomogram and a ${ }^{131} \mathrm{I}$-meta-iodobenzylguanidine (MIBG) scan were consistent with a phaeochromocytoma in the right adrenal. A $3 \mathrm{~cm}$ in diameter phaeochromocytoma was later removed and confirmed histologically.

She made an uneventful recovery and was discharged home, normotensive and receiving no medication. She has had no further episodes of palpitations up to the time of writing.

\section{Case 2}

A 37 year old man presented with headaches, anxiety, and labile hypertension. The results of the biochemical investigations for phaeochromocytoma are shown in tables 1 and 2. An abdominal computed tomogram was normal but an MIBG scan showed increased isotope uptake in the midline anterior to the lumbo-sacral junction.

Correspondence to:

Dr C Weinkove

Accepted for publication

7 October 1992 
Table 1 Urinary catecholamine and metabolite excretion ( $\mu$ mol/24 h)

\begin{tabular}{|c|c|c|c|c|c|c|c|c|}
\hline \multirow[b]{3}{*}{ Date } & \multicolumn{3}{|c|}{ Free catecholamines } & \multicolumn{5}{|l|}{ Metabolites } \\
\hline & $\begin{array}{l}\text { Noradrenaline } \\
\text { (Upper limit of }\end{array}$ & $\begin{array}{l}\text { Adrenaline } \\
\text { ence ranges) }\end{array}$ & $\begin{array}{l}\text { Total } \\
\text { catecholamines }\end{array}$ & Normetadrenaline & $\begin{array}{l}\text { Metadrenaline } \\
\text { metadrenalines }\end{array}$ & Total & $H M M A$ & $H V A$ \\
\hline & $1 \cdot 0$ & $0 \cdot 2$ & $1 \cdot 20$ & $3 \cdot 0$ & $2 \cdot 0$ & $5 \cdot 0$ & 35 & 40 \\
\hline $\begin{array}{l}\text { Case } 1 \\
21 / 10 / 90\end{array}$ & \multicolumn{8}{|c|}{ Emergency laparotomy } \\
\hline $13 / 11 / 90$ & $0 \cdot 38$ & $0 \cdot 18$ & 0.56 & $\star 3 \cdot 30$ & $\star 11 \cdot 4$ & ${ }^{\star} 14 \cdot 7$ & \multirow{2}{*}{$\begin{array}{l}\text { Insufficient } \\
\text { specimen } \\
\star 56 \\
\star 49\end{array}$} & \multirow{2}{*}{$\begin{array}{l}\text { Insufficien } \\
\text { specimen } \\
4 \\
17\end{array}$} \\
\hline $\begin{array}{l}21 / 11 / 90 \\
12 / 02 / 91 \\
27 / 02 / 91\end{array}$ & $\begin{array}{l}0.63 \\
0.47\end{array}$ & $\begin{array}{l}\star 0.59 \\
0.07\end{array}$ & $\begin{array}{l}{ }^{\star} 1.22 \\
0.54 \\
\text { Right adrenaled }\end{array}$ & $\begin{array}{r}2 \cdot 20 \\
1 \cdot 46 \\
n y\end{array}$ & $\begin{array}{r}\star 7 \cdot 7 \\
\star 3.9\end{array}$ & $\begin{array}{r}\star 9.9 \\
\star 5.4\end{array}$ & & \\
\hline $30 / 06 / 91$ & $0 \cdot 15$ & 0.03 & $0 \cdot 18$ & $0 \cdot 77$ & $0 \cdot 6$ & $1 \cdot 4$ & 20 & 15 \\
\hline $\begin{array}{l}\text { Case 2 } \\
31 / 12 / 91 \\
14 / 01 / 92\end{array}$ & $\begin{array}{l}\star 3.12 \\
\star 3.22\end{array}$ & $\begin{array}{l}0 \cdot 14 \\
0 \cdot 20\end{array}$ & $\begin{array}{l}\star 3.26 \\
\star 3.42\end{array}$ & $\begin{array}{l}2 \cdot 9 \\
\star 4 \cdot 12\end{array}$ & $\begin{array}{l}<0.5 \\
<0.5\end{array}$ & $\begin{array}{l}2 \cdot 90 \\
4 \cdot 12\end{array}$ & & \\
\hline
\end{tabular}

Both urine specimens from case 2 were collected before removal of the phaeochromocytoma. Urinary free dopamine excretion, not reported, was measured in all specimens and was within normal limits $(<3.0 \mu \mathrm{mol} / 24 \mathrm{~h})$. Note that the upper limit of the reference ranges were derived from a hypertensive population without phaeochromomytoma, with abnormal results indicated by an asterisk.

HMMA = 4-hydroxy-3-methoxy mandelic acid; HVA = homovanillic acid.

A $5 \times 4 \mathrm{~cm}$ phaeochromocytoma was subsequently removed from the lower end of the para-aortic sympathetic chain, effectively curing the patient's hypertension and headaches.

\section{Discussion}

The first patient with a phaeochromocytoma presented with retroperitoneal haemorrhage, which is unusual but well documented. ${ }^{1}$ The correct diagnosis was not suspected until the patient had undergone emergency laparotomy, which could have been fatal in a patient with a catecholamine producing tumour. ${ }^{3}$

In retrospect the four year history of episodic palpitations and breathlessness were probably manifestations of the underlying condition, because these symptoms have not recurred since removal of the tumour. This case illustrates the "diagnosis gap" which can occur between the onset of symptoms and confirmation of phaeochromocytoma. ${ }^{6}$

It was disappointing, but not surprising in the light of our past experience, ${ }^{5}$ to find normal plasma catecholamine concentrations in the preoperative blood of this patient. However, it was a matter of great concern to find that measurement of urinary free catecholamines failed to detect any abnormality in two of three urine collections from this patient, while the urinary excretion of the

Table 2 Plasma catecholamine concentrations (nmol/l) with upper limit of reference range, derived from hypertensive non-phaeochromocytoma population

\begin{tabular}{|c|c|c|c|}
\hline \multirow[b]{3}{*}{ Date } & Noradrenaline & Adrenaline & Dopamine \\
\hline & \multicolumn{3}{|c|}{ Upper limit of reference range } \\
\hline & 6 & $1 \cdot 0$ & $1 \cdot 0$ \\
\hline \multirow{9}{*}{$\begin{array}{l}\text { Case } 1 \\
26 / 02 / 91 \\
27 / 02 / 91 \\
05 / 03 / 91 \\
27 / 06 / 91 \\
\text { Case } 2 \\
14 / 01 / 92 \\
\text { Resting } \\
5 \text { min after } \\
\text { exercise } \\
10 \text { min after } \\
\text { exercise }\end{array}$} & & & \\
\hline & - & \multicolumn{2}{|c|}{$\begin{array}{l}0.76 \quad 0.57 \\
\text { Right adrenalectomy }\end{array}$} \\
\hline & $3 \cdot 3$ & $<0.2$ & 0.76 \\
\hline & $4 \cdot 1$ & $<0.2$ & $<0 \cdot 2$ \\
\hline & & & \\
\hline & 17 & $2 \cdot 1$ & $<0 \cdot 2$ \\
\hline & 26 & $2 \cdot 2$ & $<0.2$ \\
\hline & & & \\
\hline & 41 & $3 \cdot 0$ & $<0.2$ \\
\hline
\end{tabular}

Only preoperative plasma samples were available from case 2 . metabolites (the metadrenalines and HMMA) were consistently increased.

Sinclair $e t \mathrm{al}^{7}$ have recently reported normal urinary free catecholamines in a patient with phaeochromocytoma and clearly documented episodic hypertension. Unfortunately, urinary catecholamine metabolites were not measured in their patient. Peaston measured catecholamines and their metabolites in the urine of 28 surgically confirmed phaeochromocytomas using specific HPLC methods. ${ }^{8}$ Total metadrenaline excretion was increased in all their urine collections; urinary catecholamine excretion was abnormal in $98 \%$, but HMMA was raised in only $25 \%$. In a recent study of 21 phaeochromocytoma patients it was reported that increased excretion of either adrenaline or noradrenaline gave $100 \%$ diagnostic sensitivity. ${ }^{9}$ Equal sensitivity was attained by the excretion of either metadrenaline or normetadrenaline, at the cost of reduced specificity. Measurement of total catecholamines (the sum of noradrenaline and adrenaline) and total metadrenalines (the sum of normetadrenaline and metadrenaline) was less sensitive than the constituents taken separately. HMMA had the lowest sensitivity (71\%).

Experience from the first case and the reports cited above would lead us to conclude that the urinary excretion of catecholamine metabolites should replace the measurement of the free catecholamine excretion in the diagnosis of phaeochromocytoma. However, complete reliance on urinary total metadrenaline excretion would have caused us to miss the phaeochromocytoma in the second patient, who presented with the more classic features of phaeochromocytoma-headaches, anxiety attacks, and labile hypertension. Despite this strong clinical evidence of a phaeochromocytoma, the combination of normal metabolite excretion and a negative computed tomogram would have led to a misdiagnosis. Fortunately, we continued to measure free catecholamines which confirmed the diagnosis of the tumour.

As a result of our experience, we now measure both free catecholamines and their 3-methoxy amine metabolites, normeta- 
drenaline and metadrenaline, on every 24 hour urine collection from patients with suspected phaeochromocytoma. This is relatively straightforward using the Gilson ASTED (Automated Sequential Trace Enrichment of Dialysates) system (Anachem, Luton, Beds, England) which permits simultaneous measurement of catecholamines and these metabolites on the same specimen, with minimal sample preparation and no alteration to the assay conditions. ${ }^{10}$ Measurement of the unconjugated (free) catecholamines requires no sample preparation. A preliminary acid hydrolysis of the urine by incubation in a boiling water bath for 60 minutes is all that is required for the measurement of normetadrenaline and metadrenaline, which are excreted as their sulphate and glucuronide conjugates. To date, we have had no problems with drug interference in either assay and there is no need for patients to avoid specific foods or to discontinue their medication before urine collection.

Several urine collections should be analysed when there are good clinical grounds for suspecting a phaeochromocytoma. Though not to be generally encouraged, it may occasionally be clinically justified to proceed to computed tomogram and MIBG scanning in the face of negative biochemistry. Phaeochromocytomas are commonly missed; so there is an argument for screening all hypertensive patients. Any diagnostic test, as well as being cheap, will have to be both highly sensitive and specific. It is apparent from the two cases presented here that the measurement of urinary excretion of both the parent catecholamines as well as their metabolites are needed to make this important diagnosis.

On the basis of our two cases and the above reports, ${ }^{6-911}$ the following conclusions may be drawn:
1 Neither urinary free catecholamine excretion nor plasma catecholamine concentrations are acceptable as the sole biochemical test for phaeochromocytoma.

2 It is important to measure and report urinary excretion of the individual catecholamines, noradrenaline and adrenaline and the individual metabolites, normetadrenaline and metadrenaline.

3 Plasma catecholamine measurement is a less sensitive test than the urinary excretion of catecholamines and their metabolites.

4 Clinicians should be encouraged to consider the diagnosis of phaeochromocytoma more frequently.

We are grateful to Mr. Fraser Davidson of the Biochemistry Department, Crosshouse Hospital, Kilmarnock, for the HMMA and HVA analysis.

1 Manger WM, Gifford RWJr. Phaeochromocytoma. New York: Springer-Verlag, 1977.

2 Tucker RM, Labarthe DR. Frequency of surgical treatment for hypertension in adults at the Mayo clinic from 1973 through 1975. Mayo Clinic Proc 1977;52:549-55.

3 Sutton MG, Sheps SG, Lie JT. Prevalence of clinically unsuspected phaeochromocytoma. Review of a 50 year autopsy series. Mayo Clin Proc 1981;56:354-60.

4 Pillai DN, Earl JW, Duncan MW, et al. Biogenic amines: significance, measurement and clinical applications. Clin Biochem Rev 1991;12:14-21.

5 Weinkove C. ACP Broadsheet No 127. Measurement of catecholamines and their metabolites in urine. 7 Clin Pathol 1991; 44:269-75.

6 Engelman K. Principles in the diagnosis of phaeochromocytoma. Bull N Y Acad Med 1969;45:851-8.

7 Sinclair D, Shenkin A, Lorimer AR. Normal catecholamine production in a patient with a paroxysmally secreting phaechromocytoma. Ann Clin Biochem 1991; 28:417-19.

8 Peaston RT. The biochemical diagnosis of phaeochromocytoma. In: Martin SM, Halloran SP, eds. Proceedings of Associates, 1991:89.

9 Graham P, Smythe GA, Edwards G, Lazarus L. The selection of catecholamines and their metabolites in the diagnosis of phaeochromocytoma. Clin Biochem Rev 1991;12:98.

10 Green B, Cooper JDH, Tumell DC. An automated method for the analysis of urinary free catecholamines method for the analysis of urinary free catecholamines phy. Ann Clin Biochem 1989; 26:361-7.

11 Smythe GA, Edwards G, Graham P, Lazarus L Biochemical diagnosis of pheochromocytoma by simultaneous measurement of epinephrine and norepinephrine. Clin Chem 1992;38:486-92. 